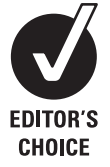

\section{See Editorial p 611}

${ }^{1}$ Cardiology Division and Clinical Analysis Laboratory, A Cardarelli Hospital, Naples, Italy;

${ }^{2}$ Cardiology Division, 2nd University of Naples, V Monaldi Hospital, Naples, Italy;

${ }^{3}$ Cardiology Division, Clinica Villa dei Fiori, Acerra, Naples, Italy;

${ }^{4}$ Health Sciences System of the Nevada System of Higher Education, Las Vegas, Nevada, USA

\section{Correspondence to:}

Dr Giovanni Gaeta, Cardiology Division and Clinical Analysis Laboratory, A Cardarelli Hospital, Napoli, Italy; giovanni.gaeta@ tin.it

Accepted 9 January 2009 Published Online First 23 January 2009

\title{
Early increase of carotid intima-media thickness in children with parental history of premature myocardial infarction
}

\author{
S Barra, ${ }^{1}$ G Gaeta, ${ }^{1}$ S Cuomo, ${ }^{1,2}$ P Guarini, ${ }^{3}$ M C Foglia, ${ }^{1}$ G Capozzi, ${ }^{2}$ C Materazzi, \\ M Trevisan ${ }^{4}$
}

\begin{abstract}
Background/objective: Increased carotid intima-media thickness (IMT) is an early manifestation of atherosclerosis. Our group has previously demonstrated that a parental history of premature myocardial infarction (PHPMI) is associated with an increase in carotid IMT in children-adolescents (mean age 13 years) and young adults (mean age 24 years). The aim of the present study was to evaluate if carotid structural changes are detectable in young children with PHPMI.
\end{abstract}

Methods: 26 healthy children $(9$ males and 17 females; 5-12 years, mean age 9.1 (2.5) years) with PHPMI and 26 age-matched (plus or minus 1 year), sex-matched and body mass index-matched (BMI; plus or minus $20 \%$ ) control subjects were enrolled in the study. They underwent high resolution B-mode ultrasonographic evaluation of common carotid artery IMT. Lipid profile, resting blood pressure and BMI were also evaluated.

Results: Compared to controls, subjects with PHPMI had increased IMT of common carotid arteries (mean of combined sites: $0.444(0.076) \mathrm{mm}$ versus 0.382 (0.062) $\mathrm{mm}$ in controls, $p=0.001)$. Offspring of coronary patients showed an unfavourable lipid profile compared to controls; however, the association between a PHPMI and carotid IMT was independent of lipids, apolipoproteins and other traditional risk factors.

Conclusions: Vascular structural changes are detectable in subjects with PHPMl at a young age and occur independently of several traditional cardiovascular risk factors.

Atherosclerosis starts during childhood. ${ }^{1-3}$ Epidemiological studies have shown that several factors, which influence disease progression could already be identified in the first decade of life, long before the build-up of plaque results in signs or symptoms of cardiovascular disease. ${ }^{45}$ Although a parental history of premature coronary artery disease has been recognised as a risk factor for heart disease, it is not clear when cardiovascular abnormalities can be identified by non-invasive methods in individuals with such history. Detection of vascular atherosclerotic changes in early childhood could help identify high-risk subjects and provide additional motivation for early-intervention strategies, focused at reducing modifiable risk factors such as obesity, hyperlipidaemia, high blood pressure and cigarette smoking. ${ }^{3}$

High-resolution B-mode ultrasound has proved to be a reliable non-invasive method to measure carotid artery intima-media thickness (IMT). ${ }^{6}$ We have already described, and other authors have confirmed, an increase of IMT in children-adolescents and young adults with parental history of premature myocardial infarction (PHPMI).-9 In this study we investigate whether early vascular anatomical changes can be detected in these children at even younger ages (age $5-12$ years).

\section{METHODS}

\section{Subjects}

Twenty-six healthy children (age 5-12 years, mean 9.1 years) with PHPMI were enrolled in the study (PHPMI-positive; 9 males, 17 females). All parents showed clinical evidence of acute myocardial infarction at or before the age of 50 years (mean age when infarction occurred: 38.9 (5.6) years). Twenty-four children had a paternal history of premature myocardial infarction while two had a maternal history. These children were recruited among offspring of patients hospitalised at Cardarelli Hospital, Naples, Italy, between July 1997 and July 2003. Twenty-six children agematched (plus or minus 1 year), sex-matched and body mass index-matched (BMI; plus or minus $20 \%$ ), without family history of coronary artery disease (including established or suspected myocardial infarction and angina pectoris) served as controls (PHPMI-negative). They were offspring of patients who had been admitted to the same hospital for a wide spectrum of acute illnesses unrelated to coronary artery disease or diabetes. This study complies with the Declaration of Helsinki. Parents gave written informed consent to the study, which was approved by the local committee on ethical practice. Six of the 26 children $(23 \%)$ had been included in our previous reports. ${ }^{78}$

\section{Cardiovascular risk factor assessment}

Measurements were made in the hospital, according to standardised protocols. All participants' parents completed a standardised questionnaire designed to obtain information about family history of vascular disease and/or diabetes. BMI was calculated as the ratio of weight in kilograms to height in square metres. Body surface area (BSA) was calculated using a modified DuBois and DuBois formula: BSA $\left(\mathrm{m}^{2}\right)=0.007184 \times$ height $(\mathrm{cm})^{0.725} \times$ weight $(\mathrm{kg})^{0.425}$. Blood pressure was measured twice on one occasion, 1 minute apart, with the participant seated, and after 5 minutes of rest (with the use of a standard mercury sphygmomanometer). Pulse pressure was calculated as systolic minus diastolic blood pressure. 


\section{Laboratory methods}

Blood samples were collected by venepuncture after 14 hours of fasting. Plasma concentrations of total cholesterol and triglycerides were measured using enzymatic colorimetric procedures (BM/Hitachi 747, Boehringer Mannheim Italia, Monza, Italy). High-density lipoprotein (HDL) and low-density lipoprotein (LDL) cholesterol concentrations were determined by agarose gel electrophoresis (Helena Laboratories, Assago, Italy). Serum apolipoprotein (Apo) A-I, Apo-B and lipoprotein (a) (Lp(a)) levels were determined by immunonephelometric methods (BNA II, Istituto Behring, Scoppito, Italy). Intra-assay technical errors were $2.9 \%$ for total cholesterol, $3.6 \%$ for triglycerides, 6.7\% for HDL-cholesterol and 6.9\% for LDL-cholesterol, 1\% for Apo A-I, $1.5 \%$ for Apo-B and $1.8 \%$ for $\mathrm{Lp}(\mathrm{a})$. Interassay coefficients of variation during the study were $3.1 \%$ for total cholesterol, 3.9\% for triglycerides, $7.9 \%$ for HDL-cholesterol, $8.0 \%$ for LDL-cholesterol, 3.3\% for Apo A-I, 4.6\% for Apo-B and $5 \%$ for $\operatorname{Lp}(\mathrm{a})$.

\section{High resolution carotid ultrasound}

High resolution carotid ultrasonographic studies were performed with a Hewlett Packard Sonos 2000, with a 7.5-MHz transducer (Hewlett-Packard, Seattle, WA, USA). The study protocol involved scanning of the far wall of common carotid arteries in the distal $1.0 \mathrm{~cm}$. The crest at the origin of the bifurcation was used as anatomical landmark to identify the segment to be visualised. In each examination the sonographer used three different scanning angles (anterior, lateral and posterior) to record the greatest IMT. Scans were recorded for offline analysis. One expert reader, who was unaware of subject's family history (PG), reviewed the scans and performed IMT quantitative measurements using the digital caliper of the ultrasound machine. For each side, the reader selected three different end-diastolic frames that contained the maximum IMT. The three calculated IMT values were averaged to give the mean common carotid IMT for each side, which is presented in the analysis. In a previous study, we assessed the intra-observer reproducibility of the same author who made carotid IMT measurements in the present study. ${ }^{7}$ The mean absolute difference in IMT in 25 healthy young subjects in whom the same observer performed two carotid artery examinations 7 days apart was $0.02(0.03) \mathrm{mm}$ (coefficient of variation, $2.6 \%$ ).

\section{Statistical analysis}

Values are expressed as mean (SD). Comparisons between PHPMI-positive and PHPMI-negative subjects were made using paired Student $t$ tests for continuous variables. Because their distributions were skewed, $\mathrm{Lp}(\mathrm{a})$ and triglycerides were logarithmically transformed before analysis.

Multivariable regression analysis was used to assess the independent relation between carotid IMT and the selected variables. In addition to PHPMI the following variablessystolic blood pressure, Apo-B and $\mathrm{Lp}(\mathrm{a})$-were included because of their established association with atherosclerosis. To take into consideration the matched design of the study, we included in the model the matched pair differences instead of the absolute values of the variables.

The size of the study sample was a priori calculated according to the results reported in our previous study (childrenadolescents age group). ${ }^{8}$ A sample size of 25 subjects in each group should have an $80 \%$ statistical power to detect a significant difference in mean common carotid IMT with a two-sided $p$ value of 0.05 . All analyses were carried out using SPSS v13.0.

\section{RESULTS}

Table 1 shows the clinical characteristics of the study subjects according to their PHPMI. Age, gender and BMI (by design) were comparable. There were no significant differences in BSA, blood pressure, total cholesterol, HDL-cholesterol and LDLcholesterol, triglycerides, $\mathrm{Lp}(\mathrm{a})$ and Apo A-I between the two groups. On the contrary, PHPMI-positive children showed significant increased Apo-B concentration compared to PHPMI-negative children.

The data on carotid IMT are summarised in table 2. PHPMIpositive children had a greater IMT of the common carotid artery (mean of combined sites: $p=0.001$ ) than subjects without such history. Between the two groups, however, no

Table 1 Clinical characteristics and cardiovascular risk factors in 26 pairs of children with and without parental history of myocardial infarction

\begin{tabular}{|c|c|c|c|}
\hline & $\begin{array}{l}\text { PHPMI-positive } \\
(n=26)\end{array}$ & $\begin{array}{l}\text { PHPMI-negative } \\
(\mathrm{n}=26)\end{array}$ & p Value \\
\hline Age (years) & $9.1(2.5)$ & $9.4(2.2)$ & * \\
\hline Males & $9(34.6 \%)$ & $9(34.6 \%)$ & * \\
\hline Body mass index $\left(\mathrm{kg} / \mathrm{m}^{2}\right)$ & $20.6(3.5)$ & $20.1(2.8)$ & $*$ \\
\hline Weight (kg) & $38.5(13.5)$ & $38.6(11.0)$ & 0.939 \\
\hline Height (m) & $1.35(0.17)$ & $1.37(0.14)$ & 0.171 \\
\hline Body surface area $\left(\mathrm{m}^{2}\right)$ & $1.18(0.28)$ & $1.20(0.23)$ & 0.490 \\
\hline Systolic blood pressure (mm Hg) & $104(12.7)$ & $105(11.3)$ & 0.933 \\
\hline Diastolic blood pressure $(\mathrm{mm} \mathrm{Hg})$ & $61.0(7.8)$ & $62.7(7.8)$ & 0.437 \\
\hline Pulse pressure $(\mathrm{mm} \mathrm{Hg})$ & $43.4(12.9)$ & $41.9(10.7)$ & 0.648 \\
\hline Total cholesterol (mg/dl) & $173(26.8)$ & $163(25.4)$ & 0.200 \\
\hline HDL-cholesterol (mg/dl) & $52.6(15.0)$ & $52.1(15.2)$ & 0.896 \\
\hline LDL-cholesterol (mg/dl) & $103(23.6)$ & $92.9(21.5)$ & 0.136 \\
\hline Triglycerides (mg/dl) & $82.4(37.0)$ & $79.7(30.7)$ & 0.874 \\
\hline Lp(a) (mg/dl) & $23.4(31.5)$ & $21.2(32.1)$ & 0.358 \\
\hline Apo A-I (mg/dl) & $149(24.0)$ & $151(27.6)$ & 0.830 \\
\hline Apo-B (mg/dl) & $85.5(19.8)$ & $74.6(14.5)$ & 0.023 \\
\hline
\end{tabular}

Paired Student $t$ tests. Data are mean (SD), unless otherwise specified. *Matched by design.

$\mathrm{p}$ Values for triglycerides and $\mathrm{Lp}(\mathrm{a})$ are for log-transformed values.

Apo, apolipoprotein; HDL, high-density lipoprotein; LDL, low-density lipoprotein; Lp(a), lipoprotein(a); PHPMI-negative, negative parental history of premature myocardial infarction; PHPMI-positive, positive parental history of premature myocardial infarction. 
Table 2 Common carotid artery IMT in 26 pairs of children with and without parental history of myocardial infarction)

\begin{tabular}{llll}
\hline & $\begin{array}{l}\text { PHPMI-positive } \\
(\mathbf{n}=\mathbf{2 6})\end{array}$ & $\begin{array}{l}\text { PHPMI-negative } \\
(\mathbf{n = 2 6 )}\end{array}$ & p Value \\
\hline Mean right CCA IMT (mm) & $0.439(0.082)$ & $0.375(0.073)$ & 0.005 \\
Mean left CCA IMT (mm) & $0.448(0.086)$ & $0.388(0.073)$ & 0.010 \\
Mean combined CCA IMT (mm) & $0.444(0.076)$ & $0.382(0.062)$ & 0.001 \\
Right CCA lumen diameter (mm) & $5.065(0.671)$ & $4.933(0.549)$ & 0.484 \\
Left CCA lumen diameter $(\mathrm{mm})$ & $5.133(0.651)$ & $4.950(0.516)$ & 0.185 \\
\hline
\end{tabular}

Data are mean (SD). Paired t test $p$ values for continuous variables.

CCA, common carotid artery; IMT, intima-media thickness; PHPMI-negative, negative parental history of premature myocardial infarction; PHPMI-positive, positive parental history of premature myocardial infarction.

difference was observed for the lumen diameter of carotid arteries.

Table 3 summarises the results of multivariable regression analysis. For all variables we used the difference in matched pairs instead of the individual value. Together with Apo-B, additional independent variables were included because of their established association with carotid IMT and atherosclerosis. The analysis revealed that mean carotid IMT was significantly associated with adverse parental history, independent of subjects' Apo-B, Lp(a) and systolic blood pressure levels.

\section{DISCUSSION}

The present findings of a significant association between parental history of myocardial infarction and increased carotid IMT in children confirm and expand previous reports showing a similar association in older children/adolescents (age range 5-18 years) and young adults, ${ }^{7-9}$ and indicate that this association can be detected at a much earlier age. This subtle structural change in carotid arteries is independent of well established risk factors for cardiovascular disease.

Atherosclerosis is a slow, progressive disease resulting from complex interactions between inherited and environmental factors. It starts in childhood and manifests itself clinically much later. ${ }^{1-3}$ High-resolution B-mode ultrasonography is an accurate, non-invasive method to measure carotid artery IMT. ${ }^{6}$ Carotid intima-media measurement is considered a surrogate marker of subclinical atherosclerosis and has been demonstrated to be a powerful predictor of clinical events related to atherosclerosis. $^{10}$

Increased thickness of carotid artery intima-media has been found in subjects with risk factors for cardiovascular disease. ${ }^{11} 12$ Some of these, such as smoking and dietary habits, are mainly lifestyle factors, whereas others, such as hypertension and hyperlipidaemia, are at least in part under genetic control. As expected, our data confirm that young people with family history of premature myocardial infarction have higher concentrations of Apo-B in comparison to people without such

Table 3 Results of multivariable regression analysis for mean common carotid intima-media thickness difference in matched pairs.

\begin{tabular}{lllr}
\hline Independent variable & $\boldsymbol{\beta}$ & SE & p Value \\
\hline Constant & 0.000 & 0.012 & 0.977 \\
PHPMI & 0.077 & 0.014 & $<0.001$ \\
Apo-B & 0.001 & 0.001 & 0.021 \\
Systolic blood pressure & 0.001 & 0.001 & 0.279 \\
Lp(a) & 0.000 & 0.000 & 0.593 \\
\hline
\end{tabular}

Dependent variable, difference in matched pairs for mean common carotid artery IMT. Independent variables, difference in matched pairs for each of the variable.

The mean value of combined sides was used for carotid IMT.

Apo, apolipoprotein; IMT, intima-media thickness; Lp(a): lipoprotein(a). history, ${ }^{4}$ but also suggest that the effect of parental history of coronary artery disease on the carotid wall is not completely explained by traditional risk factors.

The carotid wall thickening process has a complex pathogenesis that is probably influenced by the interaction of numerous environmental and genetic factors: recognition of the genetic determinants of carotid IMT and the early mechanisms leading to vascular dysfunction may provide important insight into the pathophysiology of the atherosclerosis and offer clues towards its modulation. In recent years, the weight of inherited factors on carotid IMT ("heritability") has been investigated in general population samples of different ethnicities ${ }^{13-15}$ or in selected samples of individuals with a family history of different cardiovascular diseases. ${ }^{16-20}$ Wang et a ${ }^{18}$ demonstrated, in a multivariate-adjusted model, a heritability of $40 \%$ for carotid IMT in adult offspring of patients with a history of coronary heart disease. Moskau et a $l^{19}$ found a heritability of $61 \%$ in adult offspring of patients admitted for stroke, transient ischaemic attack, coronary artery disease or other manifestations of atherosclerosis. However, to date little is known about genes affecting carotid IMT. In fact, recent studies yielded conflicting results. ${ }^{18} 21$ Current studies suggest that the weight of heritability decreases with ageing, whereas the influence of environmental and lifestyle factors increases. ${ }^{13-15}$ Thus, very young children of subjects with early-onset atherosclerotic disease may represent an ideal study population for searching potential genes implicated in the pathogenesis of atherosclerosis.

The small number of subjects included in our study and the hospital-based nature of our sample are potential important limitations. Total and LDL-cholesterol were higher in the PHPMI-positive children, although the study was not powered to detect these differences. Moreover, we acknowledge that we did not evaluate several risk factors for cardiovascular disease such as glucose metabolism, pro-thrombotic and inflammatory markers.

In summary, we have confirmed that a parental history of myocardial infarction is also independently associated with a significant increase in carotid IMT in young children. Our results show that subclinical carotid atherosclerosis may be detected early in high risk children. Further studies are needed to assess the biological mechanisms underlying the association between increased carotid IMT and parental history of myocardial infarction.

Funding: None declared.

Competing interests: None.

\section{REFERENCES}

1. Newman WP III, Wattigney W, Berenson GS. Autopsy studies in United States children-adolescents and adolescents. Relationship of risk factors to atherosclerotic lesions. Ann NY Acad Sci 1991:623:16-25. 
2. Kaprio J, Norio R, Pesonen E, et al. Intimal thickening of the coronary arteries in infants in relation to family history of coronary artery disease. Circulation 1993;87:1960-8.

3. McCrindle BW, Urbina EM, Dennison BA, et al. Drug therapy of high-risk lipid abnormalities in children and adolescents: a scientific statement from the American Heart Association Atherosclerosis, Hypertension, and Obesity in Youth Committee, Council of Cardiovascular Disease in The Young, with The Council on Cardiovascular Nursing. Circulation 2007:115:1948-67.

4. Freedman DS, Srinivasan SR, Shear CL, et al. The relation of apolipoproteins $A-I$ and $B$ in children-adolescents to parental myocardial infarction. N Engl J Med 1986;315:721-6.

5. Grobbee DE. Predicting hypertension in childhood: value of blood pressure measurement and family history. J Am Coll Nutr 1992;11(suppl):55-9.

6. Pignoli $\mathbf{P}$, Tremoli E, Poli A, et al. Intimal plus medial thickness of the arterial wall: a direct measurement with ultrasound imaging. Circulation 1986;74:1399-406.

7. Gaeta G, De Michele M, Cuomo S, et al. Arterial abnormalities in the offspring of patients with premature myocardial infarction. N Engl J Med 2000;343:840-6.

8. Cuomo S, Guarini P, Gaeta G, et al. Increased carotid intima-media thickness in children-adolescents, and young adults with a parental history of premature myocardial infarction. Eur Heart $J$ 2002;23:1345-50

9. Juonala M, Viikari JSA, Räsänen L, et al. Young adults with family history of coronary heart disease have increased arterial vulnerability to metabolic risk factors: the Cardiovascular Risk in Young Finns Study. Arterioscler Thromb Vasc Biol 2006:26:1376-82

10. O'Leary DH, Polak JF, Kronmal RA, et al. Carotid-artery intima and media thickness as a risk factor for myocardial infarction and stroke in older adults. N Engl J Med 1999:340:14-22

11. Heiss G, Sharrett AR, Barnes R, et al. Carotid atherosclerosis measured by B-mode ultrasound in populations: associations with cardiovascular risk factors in the ARIC study. Am J Epidemiol 1991:134:250-6.
12. O'Leary DH, Polak JF, Kronmal RA, et al. Distribution and correlates of sonographically detected carotid artery disease in the Cardiovascular Health Study. The CHS Collaborative Research Group. Stroke 1992;23:1752-60.

13. North KE, MacCluer JW, Devereux RB, et al, The Strong Heart Family Study. Heritability of carotid artery structure and function: the Strong Heart Family Study. Arterioscler Thromb Vasc Biol 2002;22:1698-703

14. Fox CS, Polak JF, Chazaro I, et al, Framingham Heart Study. Genetic and environmental contributions to atherosclerosis phenotypes in men and women: heritability of carotid intima-media thickness in the Framingham Heart Study. Stroke 2003:34:397-401.

15. Sayed-Tabatabaei FA, van Rijn MJ, Schut AF, et al. Heritability of the function and structure of the arterial wall: findings of the Erasmus Rucphen Family (ERF) Study. Stroke 2005;36:2351-6.

16. Xiang $\mathbf{A H}$, Azen SP, Buchanan TA, et al. Heritability of subclinical atherosclerosis in Latino families ascertained through a hypertensive parent. Arterioscler Thromb Vasc Biol 2002;22:843-8.

17. Lange LA, Bowden DW, Langefeld CD, et al. Heritability of carotid artery intimamedia thickness in type 2 diabetes. Stroke 2002;33:1876-81.

18. Wang D, Yang H, Quiñones $\mathrm{M}$, et al. A Genome-wide scan for carotid artery intimamedia thickness: the Mexican-American Coronary Artery Disease Family Study. Stroke 2005;36:540-5.

19. Moskau S, Golla A, Grothe C, et al. Heritability of carotid artery atherosclerotic lesions. An ultrasound study in 154 families. Stroke 2005;36:5-8.

20. Ryabikov A, Malyutina S, Ryabikov M, et al. Intrafamilial correlations of carotid intima-media thickness and flow-mediated dilation in Siberian population. Am J Hyperten 2007:20:248-54

21. Fox CS, Cupples LA, Chazaro I, et al. Genomewide linkage analysis for internal carotid artery intimal medial thickness: evidence for linkage to chromosome 12. Am J Hum Genet 2004;74:253-61

\section{Images in cardiology}

\section{Giant coronary artery aneurysm in Kawasaki disease}

An asymptomatic 29-year-old man was referred for evaluation 15 years after Kawasaki disease (KD) was diagnosed. The extent of cardiac involvement was unknown. An isolated giant aneurysm of the proximal left anterior descending artery (LAD) was detected with multidetector CT coronary angiography (panel). There were no stenoses.

$\mathrm{KD}$ is a systemic vasculitis of unknown aetiology with prominent cardiac involvement. It can cause coronary arteritis, myocarditis, pericarditis and valvular regurgitation. Coronary arteritis is almost invariable and causes long-term adverse arterial remodelling. Coronary aneurysms develop in about $25 \%$ of untreated patients and about $50 \%$ regress within 2 years of the acute illness. Persistent aneurysms are associated with progressive myointimal proliferation in adjacent coronary segments, with the potential to cause flow-limiting stenoses leading to ischaemic symptoms. In addition, platelet activation by high shear forces within stenotic segments and stasis in neighbouring aneurysmal segments promote coronary thrombosis, and myocardial infarction is the commonest cause of death in $\mathrm{KD}$. Aneurysm rupture is rare. ${ }^{1}$

Intravenous immunoglobulin during acute $\mathrm{KD}$ reduces aneurysm formation to $<5 \%$ of cases. Once aneurysms are established long-term aspirin is used to prevent thrombosis. Giant aneurysms (internal diameter $\geqslant 8 \mathrm{~mm}$ ) pose the greatest thrombotic risk and call for the addition of warfarin. Associated flow-limiting stenoses require percutaneous or surgical revascularisation.

Besides conventional invasive coronary angiography, transthoracic echocardiography is often used to evaluate the coronary anatomy in $\mathrm{KD}$ but was unhelpful in this case. Multidetector CT coronary angiography can be an effective

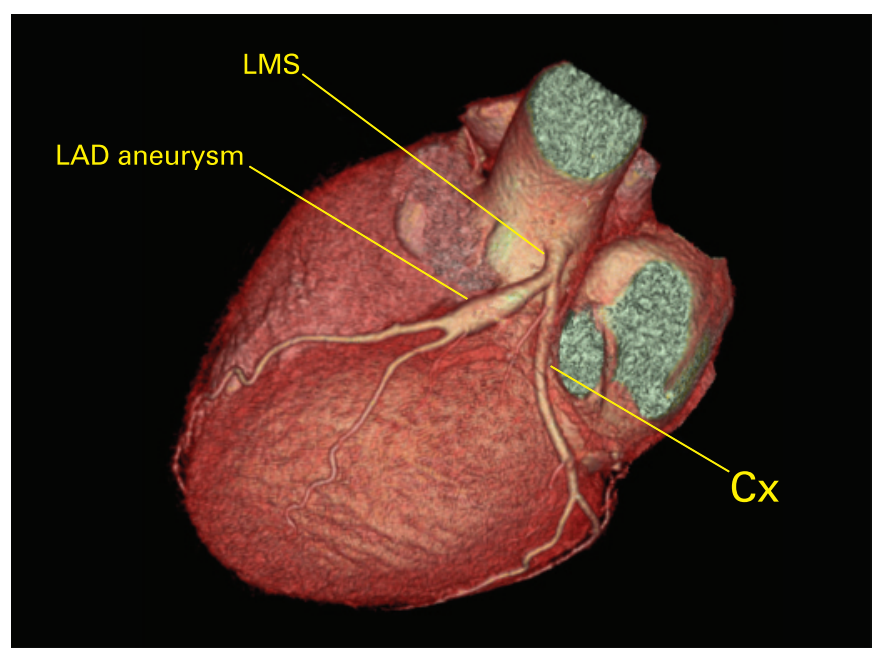

Cx, circumflex artery; LAD, left anterior descending artery; LMS, left main stem.

non-invasive alternative for establishing coronary involvement and guiding thromboprophylaxis and revascularisation in $\mathrm{KD} .{ }^{12}$

\section{O'Mahony, J M Walker, I Kayani}

c.omahony@doctors.org.uk

Heart 2009;95:645. doi:10.1136/hrt.2008.158766

\section{REFERENCES}

1. Newburger JW, Takahashi M, Gerber MA, et al. Diagnosis, treatment, and long-term management of Kawasaki disease: a statement for health professionals from the Committee on Rheumatic Fever, Endocarditis and Kawasaki Disease, Council on Cardiovascular Disease in the Young, American Heart Association. Circulation 2004;110:2747-71.

2. Aggarwala G, lyengar $\mathrm{N}$, Burke $\mathrm{S}$, et al. Kawasaki disease: role of coronary CT angiography. Int J Cardiovasc Imaging 2006;22:803-5. 